\title{
THE ISOPERIMETRIC THEOREM FOR CURVES ON MINIMAL SURFACES
}

\author{
G. D. CHAKERIAN
}

ABStract. A short proof is given for a sharpened form of the isoperimetric inequality for curves on minimal surfaces.

By following a line of development used by Sachs [7] in treating inequalities for plane curves, one can give an economical formulation to the proof of the isoperimetric theorem for curves on minimal surfaces.

Let $C$ be a smooth simple closed curve in Euclidean $n$-space, where $n \geqslant 2$. In what follows we shall assume, as can be achieved by a translation, that the centroid of arc length of $C$ is at the origin. Hence, if $x$ is the position vector, we assume

$$
\int_{C} x d s=0
$$

If we let $y(t)=x(L t / 2 \pi), 0 \leqslant t \leqslant 2 \pi$, where $L$ is the length of $C$, then (1) allows us to apply Wirtinger's inequality [1, p. 105] componentwise to obtain

$$
\int_{0}^{2 \pi}|y|^{2} d t \leqslant \int_{0}^{2 \pi}\left|\frac{d y}{d t}\right|^{2} d t
$$

from which follows

$$
\int_{C}|x|^{2} d s \leqslant \frac{L^{2}}{4 \pi^{2}} \int_{C}\left|\frac{d x}{d s}\right|^{2} d s=\frac{L^{3}}{4 \pi^{2}} .
$$

Denoting the integral on the far left-hand side of (3) by $I$, we have then an inequality of Sachs [7],

$$
L^{3}-4 \pi^{2} I \geqslant 0 .
$$

Now, with the same hypotheses as above, suppose $C$ is the boundary of a smooth orientable surface $S$ with area $A$. Then we have the area formula (see $[3],[4],[5])$

$$
\int_{C} x \cdot \nu d s=2 A+2 \iint_{S} x \cdot H d A
$$

where $\nu$ is the outward pointing unit normal to $C$ tangent to $S$, and $H$ is the mean curvature vector of $S$, which satisfies $H \equiv 0$ in case $S$ is a minimal

Received by the editors August 26, 1977.

AMS (MOS) subject classifications (1970). Primary 53A10, 52A40.

Key words and phrases. Isoperimetric inequality, minimal surface.

() American Mathematical Society 1978 
surface. In analogy to the procedure of Sachs [7] we form the vector field $D=x-(L / 2 \pi) \nu$ along $C$ and define the functional

$$
R^{2}=\frac{1}{L} \int_{C} D \cdot D d s=\frac{I}{L}-\frac{1}{\pi} \int_{C} x \cdot \nu d s+\frac{L^{2}}{4 \pi^{2}} .
$$

Substitution from (5) into (6) and rearrangement of terms gives

$$
L^{2}-4 \pi A=2 \pi^{2} R^{2}+\frac{1}{2 L}\left(L^{3}-4 \pi^{2} I\right)+4 \pi \iint_{S} x \cdot H d A .
$$

Applying (4), we have

$$
L^{2}-4 \pi A \geqslant 2 \pi^{2} R^{2}+4 \pi \iint_{S} x \cdot H d A,
$$

giving, when $H \equiv 0$, a sharpened form of the isoperimetric inequality for curves on minimal surfaces (see [2]-[6]).

When $S$ is a minimal surface, (7) yields

$$
4 \pi^{2} I-8 \pi L A+L^{3}=4 \pi^{2} L R^{2} \geqslant 0,
$$

which can be rearranged as

$$
L^{2}-4 \pi A \geqslant(4 \pi / L)(L A-\pi I) .
$$

On the other hand, (4) can be written in the form

$$
L^{2}-4 \pi A \geqslant(4 \pi / L)(\pi I-L A) .
$$

From (10) and (11) we have another sharpening of the isoperimetric inequality for curves on minimal surfaces,

$$
L^{2}-4 \pi A \geqslant(4 \pi / L)|\pi I-L A|,
$$

an inequality established in the same fashion for plane curves by Sachs [7].

\section{REFERENCES}

1. W. Blaschke, Kreis und Kugel, Leipzig, 1916; reprint, Chelsea, New York, 1949.

2. A. Dinghas, Über Minimalabbildungen von Gebieten der komplexen Ebene in einen HilbertRaum, Jber. Deutsch. Math.-Verein. 67 (1964), 43- 48.

3. K. Hanes, Isoperimetric inequalities for manifolds with boundary, J. Differential Geometry 7 (1972), 525-534.

4. C. C. Hsiung, Isoperimetric inequalities for two-dimensional Riemannian manifolds with boundary, Ann. of Math. (2) 73 (1961), 213-220.

5. R. Osserman, Isoperimetric and related inequalities, Proc. Sympos. Pure Math., vol. 27, Amer. Math. Soc., Providence, R.I., 1975, 207-215.

6. W. T. Reid, The isoperimetric inequality and associated boundary problems, J. Math. Mech. 8 (1959), 897-906.

7. H. Sachs, Ungleichungen für Umfang, Flächeninhalt und Trägheitsmoment konvexer Kurven, Acta Math. Acad. Sci. Hungar. 11 (1960), 103-115.

Department of Mathematics, University of California, Davis, California 95616 